Pacific Journal of Mathematics

LIMIT CIRCLE TYPE RESULTS FOR SUBLINEAR EQUATIONS 


\title{
LIMIT CIRCLE TYPE RESULTS FOR SUBLINEAR EQUATIONS
}

\author{
JOHN R. GRAEF
}

\begin{abstract}
Recently there has been an interest in obtaining integrability criteria for solutions of nonlinear differential equations similar in nature to those known for linear equations. In the classic paper on the subject, $H$. Weyl classified the second order linear differential equation
\end{abstract}

$$
\left(a(t) x^{\prime}\right)^{\prime}+q(t) x=0
$$

as being of the limit circle type if all its solutions are square integrable, i.e.,

$$
\int^{\infty} x^{2}(u) d u<\infty
$$

otherwise the equation was said to be of the limit point type. In this paper we discuss extensions of the limit point-limit circle classification to forced second order nonlinear equations of the type

$$
\left(a(t) x^{\prime}\right)^{\prime}+q(t) f(x)=r(t) .
$$

Throughout this paper we will assume that $a, q, r:\left[t_{0}, \infty\right) \rightarrow R$ and $f: R \rightarrow R$ are continuous, $a^{\prime}, q^{\prime} \in A C_{\mathrm{loc}}\left[t_{0}, \infty\right), a^{\prime \prime}, q^{\prime \prime} \in L_{\mathrm{loc}}^{2}\left[t_{0}, \infty\right), a(t)$ $>0, q(t)>0$ and $x f(x) \geq 0$ for all $x$. We will say that equation (2) is of nonlinear limit circle type if every solution $x(t)$ of (2) satisfies

$$
\int_{t_{0}}^{\infty} x(u) f(x(u)) d u<\infty
$$

and we will say that equation (2) is of nonlinear limit point type otherwise. (For a discussion of other possible definitions of nonlinear limit point and limit circle we refer the reader to the papers of Atkinson [1] and Graef [7].) This of course reduces to the square integrability of solutions in the case of equation (1). While some authors have discussed the nonlinear limit point-limit circle problem (see $[1,3,5,7,9,12,13,14$, 16]), the majority of the results obtained have been of the nonlinear limit point type for unforced equations. In fact only the papers of Graef [7] and Spikes $[12,13]$ contain limit circle results for equation (2). Moreover, in the papers written on the nonlinear limit point-limit circle problem to date, the case of $f(x)$ being sublinear has either been ignored completely or explicitly excluded by hypothesis from consideration. It is our purpose here to consider this case exclusively. Henceforth we consider the equation

$$
\left(a(t) x^{\prime}\right)^{\prime}+q(t) x^{\gamma}=r(t)
$$


where $a, q$ and $r$ are as above and $\gamma$ is the ratio of two odd positive integers with $0<\gamma \leq 1$.

Main results. We begin with a lemma which is needed in the proof of some of the other results in this paper. It gives sufficient conditions for all solutions of equation (3) to be bounded; the hypotheses placed on the functions $a, q$, and $r$ are compatible with those used in the remainder of the paper. For any continuous function $h$ we let

$$
h(u)_{+}=\max \{h(u), 0\} \text { and } h(u)_{-}=\max \{-h(u), 0\}
$$

so that $h(u)=h(u)_{+}-h(u)_{-}$.

LEMMA 1. If

$$
\int_{t_{0}}^{\infty}\left[(a(u) q(u))_{-}^{\prime} / a(u) q(u)\right] d u<\infty
$$

and

$$
\int_{t_{0}}^{\infty}\left[|r(u)| /(a(u) q(u))^{1 / 2}\right] d u<\infty,
$$

then all solutions of (3) are bounded.

Proof. Write equation (3) as the system

$$
\begin{gathered}
x^{\prime}=w, \\
w^{\prime}=\left(-a^{\prime}(t) w-q(t) x^{\gamma}+r(t)\right) / a(t),
\end{gathered}
$$

and define

$$
V(x, w, t)=a(t) w^{2} / 2 q(t)+x^{\gamma+1} /(\gamma+1) .
$$

Then

$$
\begin{aligned}
V^{\prime} & =r(t) w / q(t)-w^{2}(a(t) q(t))^{\prime} / 2 q^{2}(t) \\
& \leq r(t) w / q(t)+\left[(a(t) q(t))_{-}^{\prime} / a(t) q(t)\right] V .
\end{aligned}
$$

Since $|r(t) w / q(t)| \leq\left[r(t) /(a(t) q(t))^{1 / 2}\right]\left[a(t) w^{2} / 2 q(t)+1 / 2\right]$, we have

$$
\begin{aligned}
V^{\prime} \leq & {\left[r(t) /(a(t) q(t))^{1 / 2}+(a(t) q(t))_{-}^{\prime} / a(t) q(t)\right] V } \\
& +r(t) / 2(a(t) q(t))^{1 / 2} .
\end{aligned}
$$

Conditions (4), (5), and Gronwall's inequality imply that $V$ is bounded, and it follows immediately that $x(t)$ is bounded.

REMARK. Lemma 1 can be extended to include equation (2) as long as $f(x)$ satisfies $\int_{0}^{x} f(u) d u \rightarrow \infty$ as $|x| \rightarrow \infty$. 
In [7] the author introduced a new transformation which was particularly effective in handling equation (2) when $f(x)$ is superlinear. In order to make use of this same transformation and to compare the results here to those in [7], we first note that if $\gamma$ is the ratio of two odd positive integers, say $\gamma=(2 M-1) /(2 N-1)$ where $M$ and $N$ are positive integers, then we can write

$$
\gamma=2 n-1 \quad \text { where } n=(M+N-1) /(2 N-1) \text {. }
$$

We now let $\alpha=1 / 2(n+1)$ and $\beta=(2 n+1) / 2(n+1)$ and define

$$
s=\int_{t_{0}}^{t}\left[q^{\alpha}(u) / a^{\beta}(u)\right] d u \text { and } y(s)=x(t) .
$$

Under the transformation $\left(T_{n}\right)$ equation (3) becomes

$$
\ddot{y}+\alpha p(t) \dot{y}+P(t) y^{\gamma}=R(t)
$$

where ". " $=d / d s, \quad p(t)=(a(t) q(t))^{\prime} / a^{\alpha}(t) q^{\alpha+1}(t), \quad P(t)=$ $(a(t) q(t))^{\beta-\alpha}$, and $R(t)=a^{\beta-\alpha}(t) r(t) / q^{2 \alpha}(t)$. It will be convenient to write equation (6) as the system

$$
\begin{gathered}
\dot{y}=z-\alpha p(t) y \\
\dot{z}=\alpha \dot{p}(t) y-P(t) y^{\gamma}+R(t) .
\end{gathered}
$$

THEOREM 2. Suppose that condition (4) holds,

$$
\begin{aligned}
\int_{t_{0}}^{\infty} \mid\left\{(a(u) q(u))^{\prime} / a^{1 / 2}(u) q^{3 / 2}(u)\right\}^{\prime} \\
+(1 / 2-\alpha)\left[(a(u) q(u))^{\prime}\right]^{2} / a^{3 / 2}(u) q^{5 / 2}(u) \mid d u<\infty,
\end{aligned}
$$

and

$$
\int_{t_{0}}^{\infty}\left[|r(u)| /(a(u) q(u))^{\alpha}\right] d u<\infty
$$

If

$$
\int_{t_{0}}^{\infty}\left[1 /(a(u) q(u))^{n /(n+1)}\right] d u<\infty,
$$

then equation (3) is of nonlinear limit circle type, i.e., any solution $x(t)$ of (3) satisfies

$$
\int_{t_{0}}^{\infty} x^{\gamma+1}(u) d u<\infty
$$

Proof. If we define

$$
V(y, z, s)=z^{2} / 2+P(t) y^{\gamma+1} /(\gamma+1)
$$


then we have

$$
\begin{aligned}
\dot{V}(s) & =\alpha \dot{p}(t) y z+R(t) z+[\dot{P}(t) /(\gamma+1)-\alpha p(t) P(t)] y^{\gamma+1} \\
& =\alpha \dot{p}(t) y z+R(t) z .
\end{aligned}
$$

First we note that since $0<\gamma \leq 1$, we have $3<\gamma+3 \leq 4$ so $1 / 3>$ $1 /(\gamma+3)=\alpha \geq 1 / 4$. Moreover, since (4) implies that the product $a(t) q(t)$ is bounded from below away from zero, conditions (4) and (9) together imply that condition (5) holds. Hence, all solutions of (3) are bounded by Lemma 1 . Next we see that

$$
\begin{aligned}
|\dot{p}(t) y z|= & {\left[|\dot{p}(t)||y|^{(1-\gamma) / 2} /(a(t) q(t))^{(\beta-\alpha) / 2}\right] } \\
& \times\left[(a(t) q(t))^{(\beta-\alpha) / 2}|y|^{(\gamma+1) / 2}|z|\right] \\
\leq & {\left[|\dot{p}(t)||y|^{(1-\gamma) / 2} /(a(t) q(t))^{(\beta-\alpha) / 2}\right] } \\
& \times\left[(a(t) q(t))^{\beta-\alpha} y^{\gamma+1}+z^{2}\right] / 2 .
\end{aligned}
$$

Thus, if $x(t)$ is a solution of (3), then $y(s)=x(t)$ is bounded, and so

$$
\begin{aligned}
\dot{V}(s) & \leq\left[K_{1}|\dot{p}(t)| /(a(t) q(t))^{(\beta-\alpha) / 2}\right][(\gamma+1) / 2+1] V+R(t) z \\
& <\left[K_{2}|\dot{p}(t)|(a(t) q(t))^{(\beta-\alpha) / 2}+|R(t)|\right] V+|R(t)| / 2 .
\end{aligned}
$$

Now $\dot{p}(t)=p^{\prime}(t) a^{\beta}(t) / q^{\alpha}(t)$, and a computation shows that

$$
\begin{aligned}
p^{\prime}(t) /(a(t) q(t))^{(\beta-\alpha) / 2} & \\
= & (a(t) q(t))^{\prime \prime} / a^{1 / 2}(t) q^{3 / 2}(t) \\
& -\alpha\left[(a(t) q(t))^{\prime}\right]^{2} / a^{3 / 2}(t) q^{5 / 2}(t) \\
& -(a(t) q(t))^{\prime} q^{\prime}(t) / a^{1 / 2}(t) q^{5 / 2}(t) \\
= & \left\{(a(t) q(t))^{\prime} / a^{1 / 2}(t) q^{3 / 2}(t)\right\}^{\prime} \\
& +(1 / 2-\alpha)\left[(a(t) q(t))^{\prime}\right]^{2} / a^{3 / 2}(t) q^{5 / 2}(t) .
\end{aligned}
$$

Next observe that

$$
\begin{aligned}
\int_{s_{0}}^{s}|R(\tau(\xi))| d \xi & =\int_{t_{0}}^{t}\left[|R(u)| q^{\alpha}(u) / a^{\beta}(u)\right] d u \\
& =\int_{t_{0}}^{t}\left[|r(u)| /(a(u) q(u))^{\alpha}\right] d u
\end{aligned}
$$

which converges by condition (9). Hence conditions (8) and (9) together with an application of Gronwall's inequality shows that $V(s)$ is bounded. 
Therefore

$$
(a(t) q(t))^{\beta-\alpha} x^{\gamma+1}(t)=(a(t) q(t))^{\beta-\alpha} y^{\gamma+1}(s) \leq K_{3}
$$

for some constant $K_{3} \geq 0$. Condition (10) then implies that $x(t)$ must satisfy (11).

When $\gamma=1$ so that equation (3) is linear, we have that $n=1$ and $\alpha=1 / 4$. In this case, (8) is exactly the well known condition of Dunford and Schwartz [6; p. 1409]. Also, we note that the boundedness of $y(s)=$ $x(t)$ was only used on a factor of $y(s)$ when reconstructing $V$ from $\dot{V}$. When $\gamma=1$ no factoring is necessary, and the boundedness of $y(s)$ via Lemma 1 need not be invoked. Thus, in the case of linear equations, condition (4) is not needed, and our result with $r(t) \equiv 0$ would reduce to part (b) of Theorem 20 of Dunford and Schwartz [6; p. 1409].

In order to see that condition (10) is sharp, it is convenient to consider a special case of (3), namely

$$
x^{\prime \prime}+t^{\sigma} x^{\gamma}=0 \text {. }
$$

Now (10) implies that $\sigma>1+1 / n=1+2 /(\gamma+1)$ which is in agreement with what is known from asymptotic integrations of equation (12) (see, for example, Bellman [2; p. 163]).

We would also like to give conditions under which (10) is necessary for equation (3) to be of nonlinear limit circle type. The following theorem is needed in order to prove such a result.

THEOREM 3. Assume that conditions (4) and (5) hold,

$$
\int_{t_{0}}^{\infty}\left\{a(u)\left[q^{\prime}(u)\right]^{2} / q^{3}(u)\right\} d u<\infty
$$

and

$$
\int_{t_{0}}^{\infty}[|r(u)| / q(u)] d u<\infty
$$

If $x(t)$ is a nonlinear limit circle type solution of (3), then

$$
\int_{t_{0}}^{\infty}\left\{a(u)\left[x^{\prime}(u)\right]^{2} / q(u)\right\} d u<\infty .
$$

Proof. Let $x(t)$ be a nonlinear limit circle type solution (3); then $x(t)$ is bounded by Lemma 1 . Since $\left(a(t) x^{\prime}\right)^{\prime} x=\left(a(t) x^{\prime} x\right)^{\prime}-a(t)\left[x^{\prime}\right]^{2}$, a 
multiplication of equation (3) by $x(t) / q(t)$ and an integration by parts yields

$$
\begin{aligned}
& a(t) x^{\prime}(t) x(t) / q(t)-a\left(t_{1}\right) x^{\prime}\left(t_{1}\right) x\left(t_{1}\right) / q\left(t_{1}\right) \\
& \quad+\int_{t_{1}}^{t}\left[a(u) x^{\prime}(u) x(u) q^{\prime}(u) / q^{2}(u)\right] d u+\int_{t_{1}}^{t} x^{\gamma+1}(u) d u \\
& \quad-\int_{t_{1}}^{t}\left\{a(u)\left[x^{\prime}(u)\right]^{2} / q(u)\right\} d u=\int_{t_{1}}^{t}[x(u) r(u) / q(u)] d u
\end{aligned}
$$

for any $t_{1} \geq t_{0}$. Now

$$
\begin{aligned}
& \left|\int_{t_{1}}^{t}\left[a(u) x^{\prime}(u) x(u) q^{\prime}(u) / q^{2}(u)\right] d u\right| \\
& \quad \leq\left[\int_{t_{1}}^{t}\left\{a(u)\left[x^{\prime}(u)\right]^{2} / q(u)\right\} d u\right]^{1 / 2}\left[\int_{t_{1}}^{t}\left\{a(u) x^{2}(u)\left[q^{\prime}(u)\right]^{2} / q^{3}(u)\right\} d u\right]^{1 / 2} \\
& \quad \leq K_{1}\left[\int_{t_{1}}^{t}\left\{a(u)\left[x^{\prime}(u)\right]^{2} / q(u)\right\} d u\right]^{1 / 2}
\end{aligned}
$$

by Schwarz's inequality, condition (13), and the fact that $x(t)$ is bounded. Again since $x(t)$ is bounded, condition (14) insures that the integral on the right-hand side of (16) converges. If $x(t)$ is not eventually monotonic, let $\left\{t_{j}\right\} \rightarrow \infty$ be an increasing sequence of zeros of $x^{\prime}(t)$. From (16) we have

$$
K_{1} H^{1 / 2}\left(t_{j}\right)+K_{2} \geq H\left(t_{j}\right)
$$

where

$$
H(t)=\int_{t_{1}}^{t}\left\{a(u)\left[x^{\prime}(u)\right]^{2} / q(u)\right\} d u .
$$

It follows that $H\left(t_{j}\right) \leq K_{3}<\infty$ for all $j$ and thus (15) holds.

If, on the other hand, $x(t)$ is eventually monotonic, then $x(t) x^{\prime}(t) \leq 0$ for large $t$ since, otherwise, (11) would be violated. Using this fact in (16) and repeating the argument used above again shows that (15) holds.

THEOREM 4. Suppose that conditions (4), (8) and (13) hold, and

$$
\int_{t_{0}}^{\infty}\left\{\left[(a(u) q(u))^{\prime}\right]^{2} / a(u) q^{3}(u)\right\} d u<\infty
$$

If

$$
\int_{t_{0}}^{\infty}\left[1 /(a(u) q(u))^{n /(n+1)}\right] d u=\infty
$$


then every nontrivial solution of

$$
\left(a(t) x^{\prime}\right)^{\prime}+q(t) x^{\gamma}=0
$$

is of nonlinear limit point type, i.e., no nontrivial solution of (19) satisfies (11).

Proof. Proceeding as in the proof of Theorem 2 we have $\dot{V}(s)=$ $\alpha \dot{p}(t) y z$. Now let $x(t)=y(s)$ be any nontrivial solution of (19) with $x\left(t_{1}\right)=y\left(s\left(t_{1}\right)\right)=y\left(s_{1}\right) \neq 0$. By Lemma $1 y(s)$ is bounded so $|y(s)|^{(1-\gamma) / 2} \leq K_{1}$ for some $K_{1}>0$. Hence

$$
\begin{aligned}
\dot{V}(s) \geq & -\alpha|\dot{p}(t)||y(s)|^{(1-\gamma) / 2}|y(s)|^{(1+\gamma) / 2}|z(s)| \\
\geq & -\alpha K_{1}\left[|\dot{p}(t)| /(a(t) q(t))^{(\beta-\alpha) / 2}\right] \\
& \times\left[(a(t) q(t))^{\beta-\alpha} y^{\gamma+1}(s)+z^{2}(s)\right] / 2 \\
\geq & -K_{2}\left[|\dot{p}(t)| /(a(t) q(t))^{(\beta-\alpha) / 2}\right] V .
\end{aligned}
$$

If we let

$$
H(t)=K_{2}|\dot{p}(t)| /(a(t) q(t))^{(\beta-\alpha) / 2},
$$

then we can rewrite the last inequality above as

$$
\dot{V}+H(t) V \geq 0
$$

so

$$
\left(V(s) \exp \int_{s_{1}}^{s} H(\tau(\xi)) d \xi\right)^{\bullet} \geq 0
$$

Integrating we have

$$
V(s) \exp \int_{s_{1}}^{s} H(\tau(\xi)) d \xi \geq V\left(s_{1}\right) .
$$

Since condition (8) implies that the integral on the left-hand side of the last inequality above converges and $V\left(s_{1}\right)>0$, we have

$$
V(s) \geq K_{3}>0
$$

for $s \geq s_{1}$.

If we divide both members of (20) by $(a(t) q(t))^{\beta-\alpha}$ and rewrite the left-hand side in terms of $t$, we obtain

$$
\begin{array}{rl}
a(t)\left[x^{\prime}(t)\right]^{2} / 2 & q(t)+\alpha(a(t) q(t))^{\prime} x(t) x^{\prime}(t) / q^{2}(t) \\
& +\alpha^{2}\left[(a(t) q(t))^{\prime}\right]^{2} x^{2}(t) / 2 a(t) q^{3}(t) \\
& +x^{\gamma+1}(t) /(\gamma+1) \geq K_{3} /(a(t) q(t))^{\beta-\alpha} .
\end{array}
$$


Now if $x(t)$ was a limit circle solution of (19), then

$$
\int_{t_{1}}^{\infty} x^{\gamma+1}(u) d u<\infty \text { and } \int_{t_{1}}^{\infty}\left\{a(u)\left[x^{\prime}(u)\right]^{2} / q(u)\right\} d u<\infty
$$

by (11) and (15) respectively. Also, since $x(t)$ is bounded, the integral of the third term on the left-hand side of (21) converges by condition (17). Finally by Schwarz's inequality

$$
\begin{aligned}
\left|\int_{t_{1}}^{t}\left\{(a(u) q(u))^{\prime} x(u) x^{\prime}(u) / q^{2}(u)\right\} d u\right| \\
<\left[\int_{t_{1}}^{t}\left\{\left[(a(u) q(u))^{\prime}\right]^{2} x^{2}(u) / a(u) q^{3}(u)\right\} d u\right]^{1 / 2} \\
\cdot\left[\int_{t_{1}}^{t}\left\{a(u)\left[x^{\prime}(u)\right]^{2} / q(u)\right\} d u\right]^{1 / 2},
\end{aligned}
$$

and so we see that the integral of the second term on the left-hand side of (21) converges since $x(t)$ is bounded and (17) and (15) hold. Thus, by condition (18), an integration of (21) yields a contradiction and so $x(t)$ must in fact be a limit point solution of (19).

By combining Theorems 2 and 4 we can obtain a necessary and sufficient condition for equation (19) to be of nonlinear limit circle type.

THEOREM 5. Assume that conditions (4), (8), (13), and (17) hold. Then equation (19) is of nonlinear limit circle type if and only if

$$
\int_{t_{0}}^{\infty}\left[1 /(a(u) q(u))^{n /(n+1)}\right] d u<\infty .
$$

REMARK. Theorems 4 and 5 are somewhat unsatisfactory in that the technique of proof used for Theorem 4 encounters serious difficulties when a forcing term is present. This was not the case when a similar theorem [7; Theorem 11] was proved by the author for superlinear equations. It would be of some interest to see a result to Theorem 4 for equation (3).

In [7] some relationships between nonlinear limit circle equations and the oscillation and convergence to zero of solutions were discussed. (Results of this type for linear equations can be found in the papers of Patula et al.[4, 8, 10, 11].) For example, 5-7 in [7] give sufficient conditions under which all solutions of a nonlinear limit circle type equation are oscillatory. Since those theorems were general enough to include both superlinear and sublinear equations, we refer the reader to [7] for such results. We also note that if in Theorem 2 we require that $a(t) q(t) \rightarrow \infty$ as $t \rightarrow \infty$, then all solutions of (3) converge to zero as $t \rightarrow \infty$. 
We conclude this paper with another nonlinear limit point result. For convenience of notation we define $W:\left[t_{0}, \infty\right) \rightarrow R$ by

$$
W(t)=(a(t) q(t))_{-}^{\prime} / a(t) q(t)+|r(t)| .
$$

TheOREM 6. Suppose that conditions (4), (13), and (14) hold and

$$
\int_{t_{0}}^{\infty}|r(u)| d u<\infty
$$

If

$$
\int_{t_{0}}^{\infty}[1 / a(u) q(u)] d u=\infty,
$$

then equation (3) is of nonlinear limit point type.

Proof. Using the same system used in the proof of Lemma 1 and letting $V(x, w, t)=a^{2}(t) w^{2} / 2+a(t) q(t) x^{\gamma+1} /(\gamma+1)$, we have

$$
\begin{aligned}
V^{\prime} & =a(t) r(t) w+(a(t) q(t))^{\prime} x^{\gamma+1} /(\gamma+1) \\
& \geq-|r(t)|\left(a^{2}(t) w^{2} / 2+1 / 2\right)-\left[(a(t) q(t))_{-}^{\prime} / a(t) q(t)\right] V \\
& \geq-\left[(a(t) q(t))_{-}^{\prime} / a(t) q(t)+|r(t)|\right] V-|r(t)| / 2
\end{aligned}
$$

and so

$$
\left(V(t) \exp \int_{t_{0}}^{t} W(u) d u\right)^{\prime} \geq-[|r(t)| / 2] \exp \int_{t_{0}}^{t} W(u) d u
$$

Now conditions (4) and (22) imply that

$$
\exp \int_{t_{0}}^{\infty} W(u) d u \leq K_{1}<\infty
$$

so integrating (23) we have

$$
V(t) \geq V\left(t_{0}\right) / K_{1}-\int_{t_{0}}^{t}|r(u)| d u / 2
$$

Condition (22) guarantees that there exists $K_{2}>0$ such that

$$
\int_{t_{0}}^{\infty}|r(u)| d u / 2 \leq K_{2}<\infty .
$$

Hence, if $x(t)$ is any solution of (3) such that $\left(x\left(t_{0}\right), w\left(t_{0}\right)\right)=\left(x_{0}, w_{0}\right)$ and $V\left(x_{0}, w_{0}, t_{0}\right)=V\left(t_{0}\right) \geq\left(K_{2}+1\right) K_{1}$, then

$$
V(t) \geq\left(K_{2}+1\right) K_{1} / K_{1}-K_{2}=1 \text {. }
$$

Thus

$$
\int_{t_{0}}^{t}[V(u) / a(u) q(u)] d u \geq \int_{t_{0}}^{t}[1 / a(u) q(u)] d u \rightarrow \infty
$$


as $t \rightarrow \infty$. In view of Theorem $3 x(t)$ cannot be a nonlinear limit circle solution of (3).

REMARK. If $r(t) \equiv 0$, then Theorem 6 shows that all nontrivial solutions of (3) are of nonlinear limit point type. Theorem 6 is similar to part (i) of Theorem 8 in [7]. Other variants of this theorem can also be proved; for example see part (ii) of [7; Theorem 8].

\section{REFERENCES}

1. F. V. Atkinson, Nonlinear extensions of limit-point criteria, Math. Z., 130 (1973), 297-312.

2. R. Bellman, Stability Theory of Differential Equations, McGraw-Hill, New York, 1953.

3. J. Burlak, On the non-existence of $L^{2}$-solutions of nonlinear differential equations, Proc. Edinburgh Math. Soc., 14 (1965), 257-268.

4. T. A. Burton and W. T. Patula, Limit circle results for second order equations, Monatsh. Math., 81 (1976), 185-194.

5. J. Detki, The solvability of a certain second order nonlinear ordinary differential equation in $L^{p}(0, \infty)$, Math. Balk., 4 (1974), 115-119.

6. N. Dunford and J. T. Schwartz, Linear Operators, Part II; Spectral Theory, Interscience, New York, 1963.

7. J. R. Graef, Limit circle criteria and related properties for nonlinear equations, J. Differential Equations, 35 (1980), 319-338.

8. R. C. Grimmer and W. T. Patula, Nonoscillatory solutions of forced second-order linear equations, J. Math. Anal. Appl., 56 (1976), 452-459.

9. T. G. Hallam, On the nonexistence of $L^{p}$ solutions of certain nonlinear differential equations, Glasgow Math. J., 8 (1967), 133-138.

10. W. T. Patula and P. Waltman, Limit point classification of second order linear differential equations, J. London Math. Soc., (2) 8 (1974), 209-216.

11. W. T. Patula and J. S. W. Wong, An $L^{p}$-analogue of the Weyl alternative, Math. Ann., 197 (1972), 9-28.

12. P. W. Spikes, On the integrability of solutions of perturbed nonlinear differential equations, Proc. Roy. Soc. Edinburgh Sect. A, 77 (1977), 309-318.

13. Criteria of limit circle type for nonlinear differential equations, SIAM J. Math. Anal., 10 (1979), 456-462.

14. L. Suyemoto and P. Waltman, Extension of a theorem of A. Wintner, Proc. Amer. Math. Soc., 14 (1963), 970-971.

15. H. Weyl, Über gewöhnliche Differentialgleichungen mit Singularitaten und die zugehörige Entwicklung willkürlicher Funktionen, Math. Ann., 68 (1910), 220-269.

16. J. S. W. Wong, Remark on a theorem of A. Wintner, Enseignment Math., (2) 13 (1967), 103-106.

Received August 29, 1979; Research supported by the Mississippi State University Biological and Physical Sciences Research Institute. 


\section{PACIFIC JOURNAL OF MATHEMATICS \\ EDITORS}

DONALD BABBITT (Managing Editor)

University of California

Los Angeles, CA 90024

\section{Hugo Rossi}

University of Utah

Salt Lake City, UT 84112

C. C. Moore and Arthur Ogus

University of California

Berkeley, CA 94720
J. DugundiI

Department of Mathematics

University of Southern California

Los Angeles, CA 90089-1113

R. FINN and H. SAMELSON

Stanford University

Stanford, CA 94305

ASSOCIATE EDITORS
R. ARens
E. F. BECKENBACH
B. H. NeumanN
F. WOLF
K. YoshidA (1906-1982)

\section{SUPPORTING INSTITUTIONS}

UNIVERSITY OF ARIZONA

UNIVERSITY OF BRITISH COLUMBIA

CALIFORNIA INSTITUTE OF TECHNOLOGY

UNIVERSITY OF CALIFORNIA

MONTANA STATE UNIVERSITY

UNIVERSITY OF NEVADA, RENO

NEW MEXICO STATE UNIVERSITY

OREGON STATE UNIVERSITY
UNIVERSITY OF OREGON

UNIVERSITY OF SOUTHERN CALIFORNIA

STANFORD UNIVERSITY

UNIVERSITY OF HAWAII

UNIVERSITY OF TOKYO

UNIVERSITY OF UTAH

WASHINGTON STATE UNIVERSITY

UNIVERSITY OF WASHINGTON 


\section{Pacific Journal of Mathematics}

Vol. 104, No. 1

May, 1983

Nestor Edgardo Aguilera and Eleonor Ofelia Harboure de Aguilera, On

the search for weighted norm inequalities for the Fourier transform $\ldots \ldots .1$

Jin Akiyama, Frank Harary and Phillip Arthur Ostrand, A graph and its complement with specified properties. VI. Chromatic and achromatic numbers ......................................... 15

Bing Ren Li, The perturbation theory for linear operators of discrete type . . . 29

Peter Botta, Stephen J. Pierce and William E. Watkins, Linear

transformations that preserve the nilpotent matrices .............. 39

Frederick Ronald Cohen, Ralph Cohen, Nicholas J. Kuhn and Joseph

Alvin Neisendorfer, Bundles over configuration spaces .......... 47

Luther Bush Fuller, Trees and proto-metrizable spaces . . . . . . . . . . 55

Giovanni P. Galdi and Salvatore Rionero, On the best conditions on the

gradient of pressure for uniqueness of viscous flows in the whole space . . 77

John R. Graef, Limit circle type results for sublinear equations $\ldots \ldots \ldots \ldots 85$

Andrzej Granas, Ronald Bernard Guenther and John Walter Lee,

Topological transversality. II. Applications to the Neumann problem for

$y^{\prime \prime}=f\left(t, y, y^{\prime}\right) \ldots \ldots \ldots \ldots \ldots \ldots \ldots \ldots \ldots \ldots \ldots \ldots \ldots \ldots \ldots . \ldots 5$

Richard Howard Hudson and Kenneth S. Williams, Extensions of

theorems of Cunningham-Aigner and Hasse-Evans . . . . . . . . . . . 111

John Francis Kurtzke, Jr., Centralizers of irregular elements in reductive algebraic groups

James F. Lawrence, Lopsided sets and orthant-intersection by convex

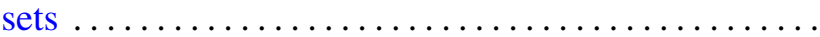

Åsvald Lima, G. H. Olsen and U. Uttersrud, Intersections of $M$-ideals and

$G$-spaces

Wallace Smith Martindale, III and C. Robert Miers, On the iterates of derivations of prime rings

Thomas H. Pate, Jr, A characterization of a Neuberger type iteration procedure that leads to solutions of classical boundary value problems

Carl L. Prather and Ken Shaw, Zeros of successive iterates of multiplier-sequence operators

Billy E. Rhoades, The fine spectra for weighted mean operators

Rudolf J. Taschner, A general version of van der Corput's difference theorem

Johannes A. Van Casteren, Operators similar to unitary or selfadjoint ones 